ARTICLE

\title{
Supramolecular copolymerization driven by integrative self-sorting of hydrogen-bonded rosettes
}

Keisuke Aratsu (10 1, Rika Takeya ${ }^{1}$, Brian R. Pauw ${ }^{2 凶}$, Martin J. Hollamby ${ }^{3 凶}$, Yuichi Kitamoto (i) ${ }^{4}$, Nobutaka Shimizu (i) ${ }^{5}$, Hideaki Takagi ${ }^{5}$, Rie Haruki ${ }^{5}$, Shin-ichi Adachi ([D ${ }^{5}$ \& Shiki Yagai (D) ${ }^{4,6 凶}$

Molecular recognition to preorganize noncovalently polymerizable supramolecular complexes is a characteristic process of natural supramolecular polymers, and such recognition processes allow for dynamic self-alteration, yielding complex polymer systems with extraordinarily high efficiency in their targeted function. We herein show an example of such molecular recognition-controlled kinetic assembly/disassembly processes within artificial supramolecular polymer systems using six-membered hydrogen-bonded supramolecular complexes (rosettes). Electron-rich and poor monomers are prepared that kinetically coassemble through a temperature-controlled protocol into amorphous coaggregates comprising a diverse mixture of rosettes. Over days, the electrostatic interaction between two monomers induces an integrative self-sorting of rosettes. While the electron-rich monomer inherently forms toroidal homopolymers, the additional electrostatic interaction that can also guide rosette association allows helicoidal growth of supramolecular copolymers that are comprised of an alternating array of two monomers. Upon heating, the helicoidal copolymers undergo a catastrophic transition into amorphous coaggregates via entropy-driven randomization of the monomers in the rosette.

\footnotetext{
${ }^{1}$ Division of Advanced Science and Engineering, Graduate School of Science and Engineering, Chiba University, 1-33 Yayoi-Cho, Inage-Ku, Chiba 263-8522, Japan. ${ }^{2}$ BAM Federal Institute for Materials Research and Testing Unter den Eichen 87, 12205 Berlin, Germany. ${ }^{3}$ School of Physical and Geographical Sciences, Keele University, Keele, Staffordshire ST55BG, UK. ${ }^{4}$ Institute for Global Prominent Research (IGPR), Chiba University, 1-33 Yayoi-Cho, Inage-Ku, Chiba 263-8522, Japan. ${ }^{5}$ Photon Factory, Institute of Materials Structure Science, High Energy Accelerator Research Organization, Tsukuba 305-0801, Japan. ${ }^{6}$ Graduate School of Engineering, Chiba University, 1-33 Yayoi-Cho, Inage-Ku, Chiba 263-8522, Japan. ${ }^{\bowtie}$ email: brian.pauw@bam.de; m.hollamby@keele.ac.uk, yagai@faculty.chiba-u.jp
} 
T he hierarchical self-assembly of macromolecular building blocks into hierarchical nanoarchitectures through the formation of oligomeric complexes is a strategy essential to living systems ${ }^{1}$. As small conformational changes in the complexes may severely affect the outcome of such hierarchical processes, biological molecular systems behave dynamically: they operate in conditions far from equilibrium and optimize component structures and functions according to environment ${ }^{2}$. This is true of not only the protein ensembles but also relatively simple one-dimensional assemblies of proteins, i.e., biological supramolecular polymers ${ }^{3-7}$. For example, two structurally related polypeptides of $\alpha$ - and $\beta$-tubulin monomer subunits complementarily form $\alpha-\beta$ heterodimer complexes through hydrophobic and electrostatic interactions ${ }^{8-11}$. These heterodimers then assemble one-dimensionally into microtubules. GTP hydrolysis within the tubulin heterodimer induces a small conformational change, triggering catastrophic microtubule disassembly $^{12}$. We envisaged that the incorporation of a molecular recognition process to preorganize polymerizable complexes of an artificial supramolecular polymer ${ }^{13-15}$ could generate smart macromolecular systems whose assembly and disassembly are controlled by the conformation of the complexes ${ }^{16,17}$. As synthetic polymers have evolved into functional materials with precise molecular sequences by controlling the kinetic reaction between monomers, similar efforts are essential in the development and practical application of supramolecular polymers.

We sought to create such an atypical artificial supramolecular polymer using simple synthetic molecules. In recent years, we have explored the self-assembly properties of a series of barbiturated $\pi$-conjugated molecules ${ }^{18-22}$. These molecules initially organize into six-membered complexes referred to as rosettes (Fig. 1a) through self-complementary hydrogen bonding. The rosettes subsequently stack through $\pi-\pi$ interactions into a supramolecular polymer with an intrinsic radius of curvature of 7-15 nm. Extending this hierarchical process has also led to supramolecular polymers with helicoidal higher-order structures $^{21,22}$. Here, we focus on molecule 1 (Fig. 1b) ${ }^{23,24}$, which triggers the development of these unique supramolecular polymers. This molecule, whose $\pi$-conjugated core is naphthalene, mainly forms uniform toroidal (circular) fibers as closed nanostructures, rather than open-ended helicoidal structures (Fig. 1c). The efficient ring-closure during the continuous stacking of the rosette complex of $\mathbf{1}$ was attributed to its small enthalpic gain through $\pi-\pi$ interactions, which prevents the supramolecular polymer chains from elongating into helicoidal structures. Previously, we have attempted to modulate the interaction between the rosettes by mixing a molecule with different substitution position or expanding the $\pi$-moiety ${ }^{25-27}$. However, mixtures of rosette-forming monomer subunits with others that contained a different $\pi$-conjugated core all exhibited narcissistic self-sorting $28-30$.

Herein, we apply a much smaller mutation to $\mathbf{1}$, by introducing a carbonyl group in the linker moiety to form $\mathbf{2}$ (Fig. 1b). It is predicted that enhancing the stacking force between rosette complexes by admixing a monomer subunit with an electronically complementary $\pi$-conjugated core might realize alternating supramolecular copolymerization ${ }^{31-34}$ : due to its modification, the $\pi$-conjugated core of $\mathbf{2}$ is electron-deficient, and can thus interact with 1 via electrostatic interactions. Mixtures of subunits 1 and $\mathbf{2}$ can randomly coassemble through hydrogen bonds at the rosette level, but at the same time, there may be an optimum unit arrangement that maximizes electrostatic interactions. We demonstrate that the competition of the random coaggregation of the two monomer subunits and the formation of an optimum rosette complex results in the autonomous progression of a supramolecular copolymerization process that selectively incorporates only one of the potential rosette complexes from the pool of various kinetically formed complexes over several days (Fig. 1e). The resulting thermodynamically stable helicoidal copolymers are thermally stable to a specific temperature, but above this transition catastrophically into a kinetic mixture of the rosette complexes due to the internal disorganization of the integrated rosette complex.

\section{Results}

Self-aggregation of 2 . The absorption maximum of monomeric 2 possessing the ester linkage in $\mathrm{CHCl}_{3}$ is hypsochromically shifted by $23 \mathrm{~nm}$ compared to $\mathbf{1}$, reflecting the reduced electron density of its naphthalene core (Supplementary Fig. 12). Although a ${ }^{1} \mathrm{H}$ NMR study of 2 in $\mathrm{CDCl}_{3}$ indicated that its hydrogen-bonding capability was almost identical to $\mathbf{1}$, atomic force microscopy (AFM) and transmission electron microscopy (TEM) images of self-assembled 2 in methylcyclohexane (MCH) exhibited only indistinct linear fibrils (Fig. 1d and Supplementary Figs. 13-16). A temperature-dependent absorption spectral analysis of $\mathbf{2}$ in $\mathrm{MCH}$ showed a hypsochromic shift of the major absorption band upon cooling, while a bathochromic shift was observed for $\mathbf{1}$ (Supplementary Fig. 17). The temperature-dependence of the degree of aggregation could be fitted with a nucleation-elongation model ${ }^{35,36}$. A van't Hoff analysis of data collected at different concentrations provided an elongation enthalpy $\left(\Delta H^{\circ}=-58 \mathrm{~kJ}\right.$ $\left.\mathrm{mol}^{-1}\right)$ that is smaller than that of $\mathbf{1}\left(\Delta H^{\circ}=-72 \mathrm{~kJ} \mathrm{~mol}^{-1}\right)$ as well as an elongation entropy $\left(\Delta S^{\circ}=-92 \mathrm{~J} \mathrm{~mol}^{-1} \mathrm{~K}^{-1}\right.$ for 2 , $\Delta S^{\circ}=-139 \mathrm{~J} \mathrm{~mol}^{-1} \mathrm{~K}^{-1}$ for 1) (Supplementary Fig. 18 and Supplementary Table 1). The very different spectroscopic and microscopic outcomes obtained upon introducing the ester group suggest that the lower electron density of the naphthalene core of $\mathbf{2}$ and/or dipole repulsion between the ester groups hamper the idiosyncratic stacking responsible for the formation of curved rosette aggregates.

Kinetic coassembly. Molecules of $\mathbf{1}$ and $\mathbf{2}$ kinetically coassembled into amorphous coaggregates when a 1:1 molar mixture of their monomers in $\mathrm{MCH}$ was cooled. Upon cooling a 1:1 mixture of $\mathbf{1}$ and 2 (hereafter denoted as $\mathbf{1 / 2}$ ) with a total concentration $\left(c_{\mathrm{t}}\right)$ of $100 \mu \mathrm{M}$ from 80 to $20^{\circ} \mathrm{C}$ at a rate of $1^{\circ} \mathrm{C} \mathrm{min}^{-1}$, the absorption spectrum of the mixture broadened considerably (Fig. $2 \mathrm{a}$ and Supplementary Fig. 19). The increased absorption observed in the red-shifted region $(450-500 \mathrm{~nm})$ is characteristic of an electronic interaction between naphthalene chromophores. Due to the aggregation-induced suppression of bond rotation in the excited state, the mixture gradually became emissive in the yellow-green region upon cooling (Fig. 2b). The cooling curve obtained by plotting the absorption at $470 \mathrm{~nm}$ as a function of temperature confirmed the coassembly of $\mathbf{1}$ and 2: the onset of the spectral change of the mixture began at a higher temperature $\left(\sim 68^{\circ} \mathrm{C}\right.$; blue dots in Fig. 2c) than that predicted by simulation that assumed narcissistic self-sorting $\left(56^{\circ} \mathrm{C}\right.$; black dots in Fig. $\left.2 \mathrm{c}\right)$. The experimental curve was fitted using the nucleation-elongation model, and the values of $\Delta H^{\circ}\left(-55 \mathrm{~kJ} \mathrm{~mol}^{-1}\right)$ and $\Delta S^{\circ}\left(-88 \mathrm{~J} \mathrm{~mol}^{-1} \mathrm{~K}^{-1}\right)$ were estimated by a van't Hoff analysis of the data collected at different concentrations (Supplementary Fig. 20). The $\Delta H^{\circ}$ value is smaller than those of $\mathbf{1}\left(-72 \mathrm{~kJ} \mathrm{~mol}^{-1}\right)$ and $\mathbf{2}\left(-58 \mathrm{~kJ} \mathrm{~mol}^{-1}\right)$, suggesting that the coassembly did not exhibit any enthalpic advantage (Supplementary Table 1). Dynamic light scattering (DLS) measurements of the freshly cooled mixture showed an average hydrodynamic diameter $\left(D_{\mathrm{H}}=15 \mathrm{~nm}\right)$ far smaller than observed for toroids of 1 and linear fibrils of 2 (Fig. 2d). In line with these observations, only amorphous coaggregates were observed in AFM and TEM images of the as-cooled fresh solution 
a

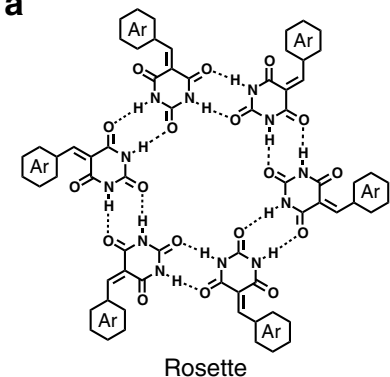

b

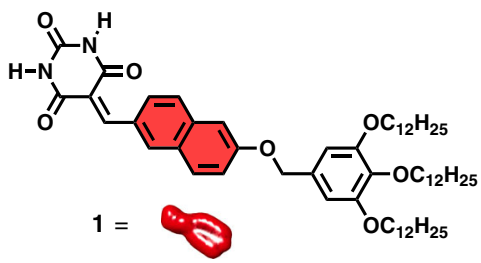

C
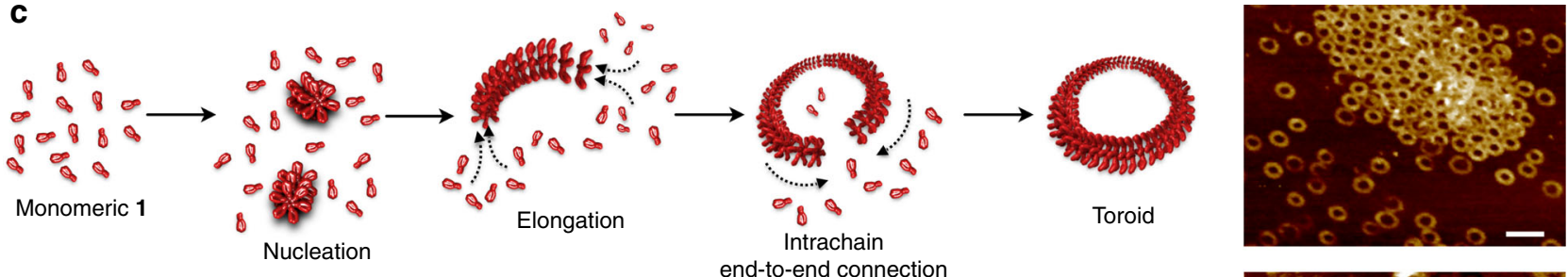

end-to-end connection

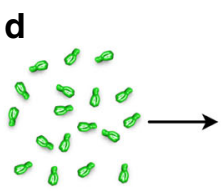

Monomeric 2
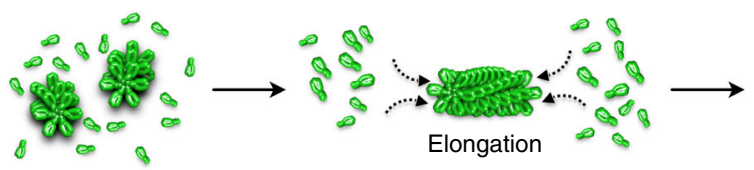

Nucleation
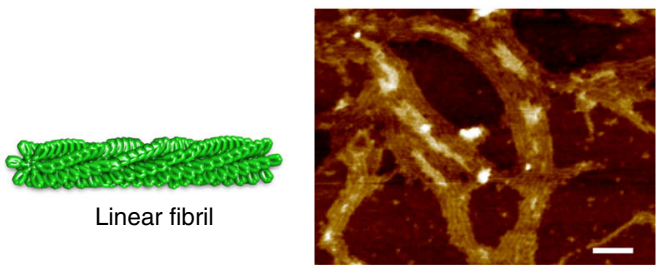

e

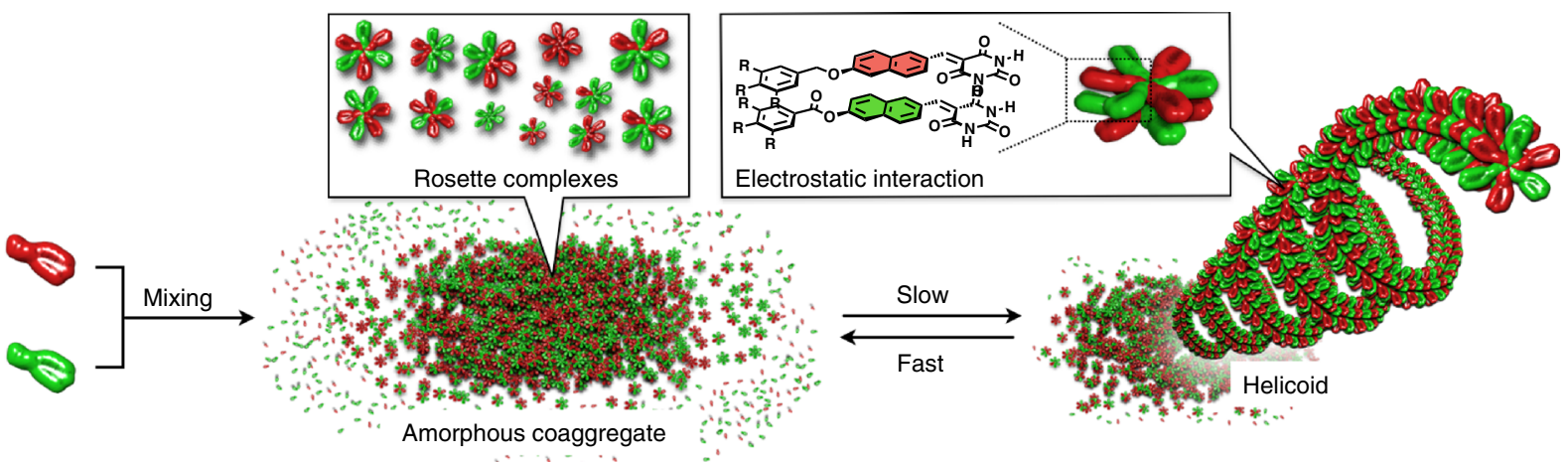

Fig. 1 Supramolecular polymerization and copolymerization. a Chemical structure of the barbituric acid rosette. $\mathbf{b}$ Chemical structure of toroid-forming molecule 1, partially modified auxiliary molecule 2. c, d Schematic representation of the supramolecular polymerization of $\mathbf{1}$ into a toroid (c) and $\mathbf{2}$ into a linear fibril (d) together with AFM images of the fibers $\left(c_{t}=250 \mu M\right)$. Scale bars, $50 \mathrm{~nm}$. e Schematic representation of the supramolecular copolymerization of $\mathbf{1}$ and $\mathbf{2}$ into a helicoid and the thermal transition from the helicoid to an amorphous coaggregate.

spin-coated onto appropriate substrates (Fig. 2f, h and Supplementary Fig. 21).

Autonomous supramolecular copolymerization. Isothermal supramolecular copolymerization of $\mathbf{1}$ and $\mathbf{2}$ occurs from the amorphous coaggregated state described above. The absorption spectra of the mixture gradually became vibronic over the course of several days at $20^{\circ} \mathrm{C}$, indicating a more uniform electronic interaction between the chromophores (Fig. 2a). A $50 \%$ attenuation of the emission was also observed (Fig. 2b) with a similar kinetic constant to that of the absorption spectral change $\left(k_{\mathrm{abs}}=0.095 \mathrm{~h}^{-1} ; k_{\mathrm{em}}=0.101 \mathrm{~h}^{-1}\right)$, suggesting a degree of charge transfer from electron-rich 1 to electron-poor 2 (Supplementary Fig. 22). These spectroscopic changes were accompanied by a gradual increase in aggregate size that reached an equilibrium value $\left(D_{\mathrm{H}}=190 \mathrm{~nm}\right)$ after $60 \mathrm{~h}$ (Fig. $2 \mathrm{e}$ ). When aliquots of the solution at different equilibration times were spin-coated onto highly oriented pyrolytic graphite (HOPG), the resulting AFM images demonstrated time-dependent growth of the helicoidal structures (Fig. 2j-q and Supplementary Figs. 23 and 24). Highresolution imaging revealed an outer helicoidal diameter of approximately $23 \mathrm{~nm}$ and pitch of approximately $5.7 \mathrm{~nm}$ (Fig. $2 \mathrm{~g}$, i). The outer diameter is slightly larger than for toroids formed by $1(20 \mathrm{~nm})$, perhaps due to the flattening of the helicoids by edgeon adsorption onto the HOPG substrate. These results suggest that the topological extension from a toroidal to a helicoidal fiber was achieved ${ }^{37}$, without synthetic extension of the aromatic core as we have done so previously ${ }^{21,22}$.

Given that much slower supramolecular copolymerization kinetics were observed upon increasing $c_{\mathrm{t}}$ (Supplementary Fig. 22), we inferred that the initially formed amorphous coaggregates are off-pathway products in the formation of the helicoids ${ }^{38-40}$. A time-dependent infrared (IR) analysis of the copolymerization revealed a remarkable shift for the two carbonyl vibrations of the hydrogen-bonded barbituric acids of $\mathbf{1}$ and $\mathbf{2}$ (Fig. 3a, b). Hence, it can be concluded that the two monomer subunits kinetically coassemble by hydrogen-bonding to form various rosette complexes, and continuously dissociate and reassemble with rearrangement of the hydrogen bonds until the enthalpy gain of 

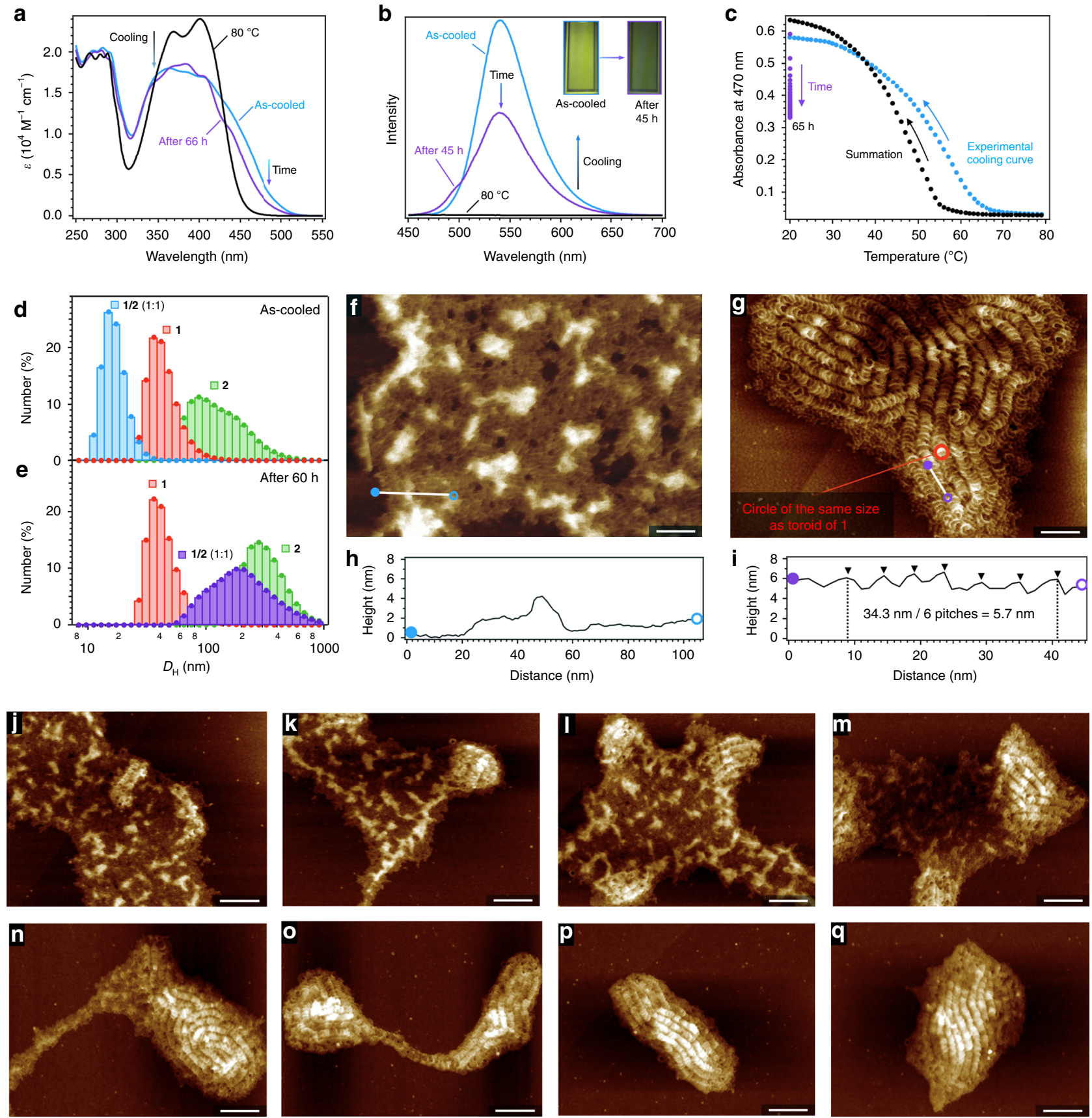

Fig. 2 Supramolecular copolymerization. a, b Absorption (a) and fluorescence emission (b) spectra of a $1: 1$ mixture of 1 and $\mathbf{2}\left(c_{t}=100 \mu \mathrm{M}\right)$ at $80^{\circ} \mathrm{C}$ (black lines) and $20^{\circ} \mathrm{C}$ (blue lines) upon cooling, and the subsequent time-dependent change after several days at $20^{\circ} \mathrm{C}$ (purple lines). Insets in $\mathbf{b}$ are photographs of the solution at $20^{\circ} \mathrm{C}$ under illumination with UV light. c Plots of the change in the absorbance intensity at $470 \mathrm{~nm}$ as a function of temperature upon cooling (blue dots), followed by the time-dependent change (purple dots). The black dots were obtained by simple summation of the separately measured cooling plots of $\mathbf{1}\left(c_{\mathrm{t}}=50 \mu \mathrm{M}\right)$ and $\mathbf{2}\left(c_{\mathrm{t}}=50 \mu \mathrm{M}\right)$. d, e DLS profiles of $\mathbf{1}\left(\mathrm{red} ; c_{\mathrm{t}}=100 \mu \mathrm{M}\right), \mathbf{2}\left(\mathrm{green} ; c_{\mathrm{t}}=100 \mu \mathrm{M}\right)$, and their $1: 1$ mixture (blue and purple; $c_{\mathrm{t}}=100 \mu \mathrm{M}$ ) in $\mathrm{MCH}$ measured immediately after cooling to $20^{\circ} \mathrm{C}(\mathbf{d})$ and after aging for $60 \mathrm{~h}$ (e). f, $\mathbf{g}$ AFM images of the amorphous coaggregates ( $\mathbf{f}$ aged for $\mathrm{O} \mathrm{h}$ ) and helicoids ( $\mathbf{g}$ aged for $31 \mathrm{~h}$ ) formed by a 1:1 mixture of $\mathbf{1}$ and $\mathbf{2}\left(\mathrm{c}_{\mathrm{t}}=100 \mu \mathrm{M}\right)$ at $20^{\circ} \mathrm{C}$. $\mathbf{h}, \mathbf{i} \mathrm{Cross}$-sectional analyses along the white lines in $\mathbf{f}$ and $\mathbf{g}$, respectively. Scale bars, $50 \mathrm{~nm}$. $\mathbf{j}-\mathbf{q}$ AFM images showing the transition of the amorphous coaggregates into helicoids. The samples were spin-coated onto highly oriented pyrolytic graphite (HOPG) substrates and aged for $0.5 \mathrm{~h} \mathrm{(j)}, 1 \mathrm{~h}(\mathbf{k}), 2 \mathrm{~h}(\mathbf{I}), 4 \mathrm{~h}(\mathbf{m}), 6 \mathrm{~h}(\mathbf{n})$, $20 \mathrm{~h}(\mathbf{0}), 24 \mathrm{~h}(\mathbf{p})$, and $72 \mathrm{~h}(\mathbf{q})$. Scale bars, $100 \mathrm{~nm}$.

the system is maximized through the electrostatic interaction between $\mathbf{1}$ and $\mathbf{2}$. The ideal monomer arrangement to maximize the enthalpy gain is integrative self-sorting 41,42 into the alternating 1:2:1:2:1:2: hydrogen-bonded rosette. These resulting complexes then stack with a rotational offset of $\sim 60^{\circ}$ to optimize the interaction between alternating $\mathbf{1}$ and $\mathbf{2}$ subunits within the complex (Fig. 1e).
Although the kinetic stability of the initially formed amorphous coaggregates is sufficiently low for their autonomous copolymerization to occur at $20^{\circ} \mathrm{C}$, this process could be further accelerated by the addition of a third component to the initial monomer mixture. Previously, we showed that $\mathbf{1}$ and its regioisomer 3 (Fig. 3c) narcissistically self-sort from kinetically formed amorphous coaggregates into toroidal and cylindrical 

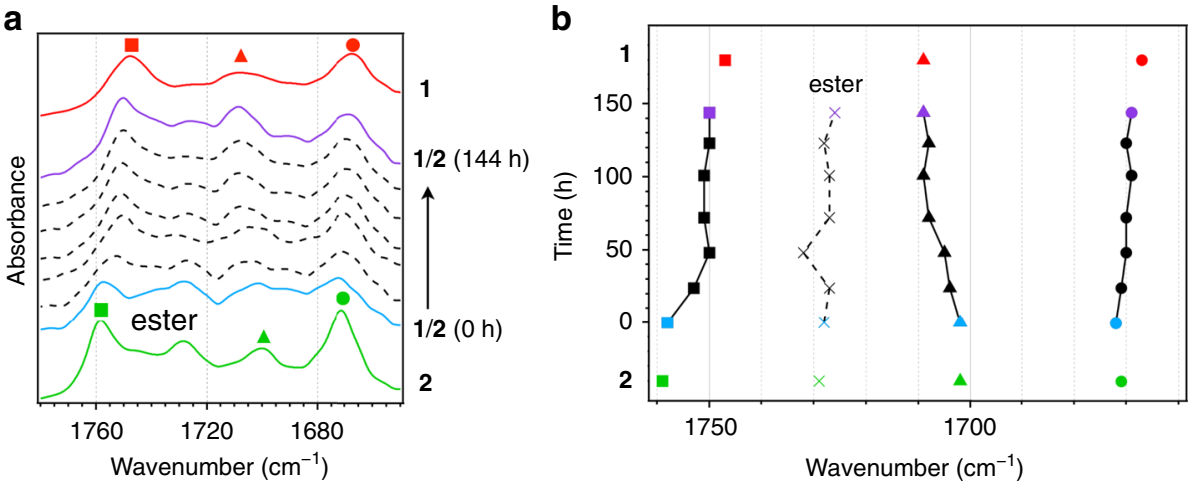

C

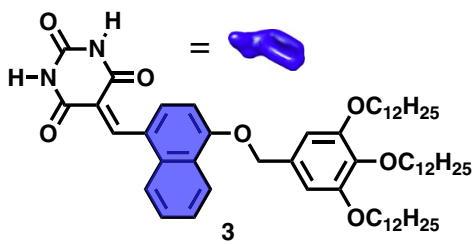

d

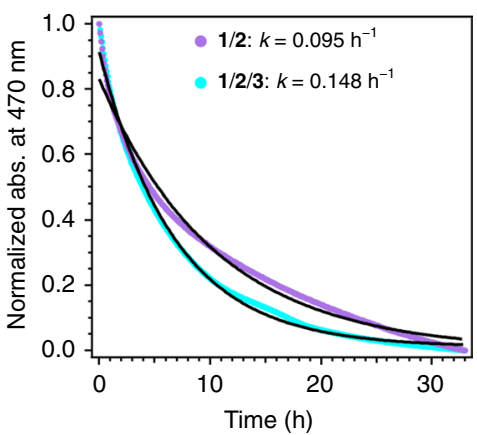

e

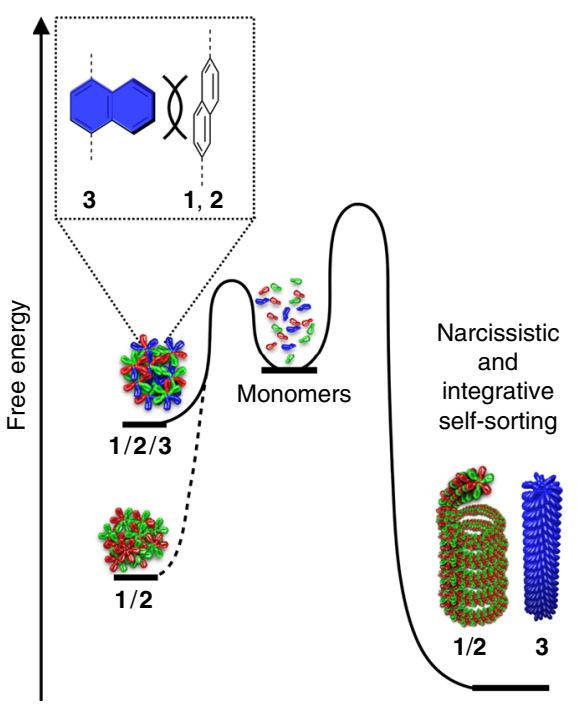

$\mathbf{f}$

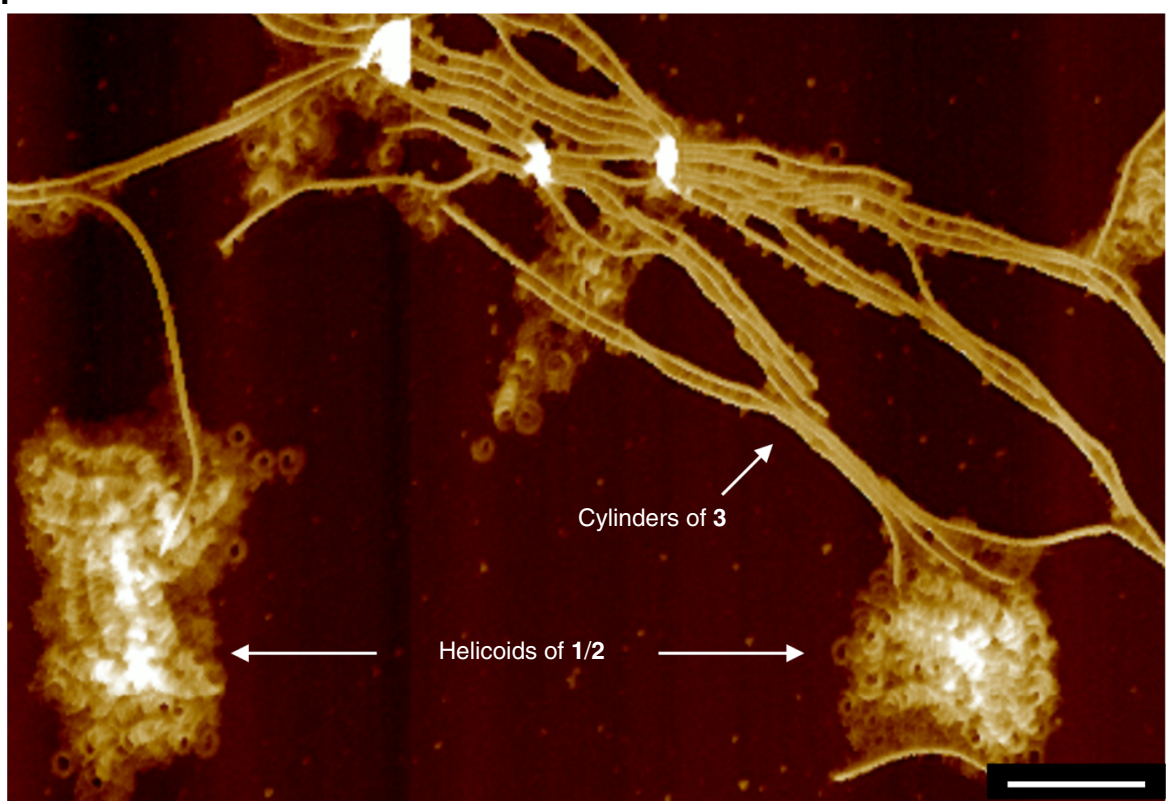

Fig. $\mathbf{3}$ IR analysis and modulation of supramolecular copolymerization. a IR spectra of toroids of $\mathbf{1}$ (red), linear fibrils of $\mathbf{2}$ (green), and a series of spectra showing the time-dependent spectral change of $\mathbf{1} / \mathbf{2}\left(c_{t}=500 \mu \mathrm{M}\right)$ from amorphous coaggregates (blue) to helicoids (purple). $\mathbf{b}$ Plots of the position of the maxima of the $\mathrm{C}=\mathrm{O}$ stretching vibrations as a function of time $(\mathrm{h})$. The colors and shapes of the symbols correspond to those in $\mathbf{a}$. $\mathbf{c} C$ hemical structure of 3. $\mathbf{d}$ Plots of the change in the normalized absorption at $470 \mathrm{~nm}$ for $\mathbf{1 / 2} \mathbf{2} \mathbf{3}$ (blue; $c_{\mathrm{t}}=150 \mu \mathrm{M}$ ) and $\mathbf{1} / \mathbf{2}$ (purple; $c_{\mathrm{t}}=100 \mu \mathrm{M}$ ) at $20^{\circ} \mathrm{C}$ after cooling. e Qualitative energy landscapes of the $\mathbf{1 / 2}$ and $\mathbf{1 / 2} / \mathbf{3}$ systems. f AFM image of $\mathbf{1 / 2 / 3}$ after $24 \mathrm{~h}$ of aging. Scale bar, $100 \mathrm{~nm}$. 
supramolecular polymers, respectively ${ }^{25}$. This narcissistic selfsorting is due to the very different molecular conformations of these regioisomers; the naphthalene ring introduced on the short axis of $\mathbf{3}$ is sterically too demanding to allow the formation of intrinsically curved rosette stacks. We thus expected that the incorporation of this sterically demanding molecule could suppress the kinetic assembly of the various rosette complexes (Fig. 3e). Upon cooling a 1:1:1 ternary mixture of $\mathbf{1 - 3}\left(\mathbf{1} / \mathbf{2} / \mathbf{3} ; c_{\mathrm{t}}=\right.$ $150 \mu \mathrm{M})$, only amorphous coaggregates were initially observed by AFM, with no trace of individual self-assemblies (Supplementary Fig. 25). An AFM image obtained after 1 day already showed not only the formation of helicoids of $\mathbf{1}$ and 2, but also that of cylindrical fibers of $\mathbf{3}$, suggesting the concerted progression of integrative and narcissistic self-sorting through dynamic molecular recognition (Fig. 3f). The kinetics of this ternary self-sorting process $\left(k_{\mathrm{abs}}=0.148 \mathrm{~h}^{-1}\right)$ were indeed much faster than that of the binary process $\left(k_{\mathrm{abs}}=0.095 \mathrm{~h}^{-1}\right.$; Fig. $\left.3 \mathrm{~d}\right)$. Similar control of kinetic effects through molecular recognition has been observed in other thermodynamic self-sorting systems ${ }^{43}$.

SAXS analysis of the supramolecular copolymerization. The growth of the helicoidal supramolecular copolymers of $\mathbf{1}$ and $\mathbf{2}$ was studied by in situ small-angle X-ray scattering (SAXS) measurements. Figure $4 \mathrm{a}$ shows the time-dependent SAXS profiles of a concentrated 1:1 mixture $\left(c_{\mathrm{t}}=500 \mu \mathrm{M}\right)$ of $\mathbf{1}$ and 2 measured after cooling. The scattered intensity, $I(Q)$ is plotted as a function of the scattering vector, $Q$. During the initial stages $(0-2.5 \mathrm{~h})$, the profiles correspond to small $(<10 \mathrm{~nm})$ size-disperse scatterers, with no visible oscillatory features. The observed increase in $I(Q)$ at low $Q\left(0.1-2 \mathrm{~nm}^{-1}\right)$, which is particularly apparent after $2.5 \mathrm{~h}$, suggests the association of these early aggregates into larger objects and may indicate an onset of supramolecular copolymerization. From $5 \mathrm{~h}$ onward, a nonperiodic oscillatory feature at $Q=0.3-1 \mathrm{~nm}^{-1}$ can be observed. Such features also appear in SAXS profiles arising from toroids of 1 and helicoids of previously reported pnp (Supplementary Figs. 26 and 27) 22,24 . As such, their presence here indicates the presence of supramolecular copolymers in solution. However, in comparison to that for pnp, here the SAXS from the helicoids of $\mathbf{1} / 2$ exhibits an additional sharp scattering peak at $Q=1.15 \mathrm{~nm}^{-1}$ $(d=5.5 \mathrm{~nm})$, which notably increases in intensity over time (inset in Fig. 4a and Supplementary Fig. 28). This peak is likely to correspond to the helicoidal pitch $(p)$ of the fibers as shown in Fig. $4 \mathrm{~g}$, which was estimated from AFM images to be $\sim 5.7 \mathrm{~nm}$ (Fig. 2i).

To verify the above assignment of the SAXS features, the scattering of a model helicoid was simulated using the SPONGE, a materials science-oriented scattering pattern calculator (Supplementary Methods). SPONGE allows the simulation of a scattering pattern from any three-dimensional solid and permits the inclusion of realistic characteristics such as polydispersity and structural dynamics. By systematically varying individual structural parameters, including $p$ (Fig. $4 \mathrm{~b}, \mathrm{~g}$ ), the center-to-center distance $(D$; Fig. $4 c, g)$, the persistence length $(n)$ defined by the turn number (Fig. 4d), and the dynamical coil motion (spring motion; Fig. 4e), their effects on the scattering pattern have been identified. In Fig. 4a, we compare the experimental data with the closest simulation result obtained using $p=5.5 \mathrm{~nm}$, a numberweighted gaussian size dispersity of $5 \%, D=16 \mathrm{~nm}$, a persistence length of 14 turns $(160 \mathrm{~nm})$ and, consistent with our previous work, an ellipsoidal wire cross-section of $6.0 \times 3.6 \mathrm{~nm}^{2}$ (flattened in the pitch direction). The overall shape of the simulation matches well with the experimental SAXS. Discrepancies in absolute intensity, particularly at mid-to-high $Q$, can be explained by (i) the unaccounted for presence of an incoherent background in the experimental SAXS, and (ii) contrast effects (the simulations assume a two-phase sharp-contrast solvent-structure, whereas the helicoidal structures have a more complex internal electron-density structure).

The more apparent pitch peak in the experimental SAXS profile of the helicoids of $\mathbf{1 / 2}$ compared to that of the previously reported unimolecular helicoids of pnp is indicative of greater stiffness in the former. A reduction in stiffness would lead to increased swinging in the $x$ and $y$ directions as well as increased motion in the $z$-direction (spring motion; Fig. 4e). In SAXS analysis, such motion manifests as a reduced persistence length ( $n$; Fig. $4 \mathrm{~d}$ ) of the fixed, uniform structure and will cause a sharp pitch peak to initially broaden and eventually become unobservable. Several reasons might account for a different stiffness: the elongation of the unimolecular helicoids of pnp was achieved by cooling, because the elongation process began at a high temperature in which the alkyl chains are in a molten state. This process is very different from that of the helicoids of $\mathbf{1 / 2}$, for which the elongation proceeds at $20^{\circ} \mathrm{C}$. At this lower temperature, the long alkyl chains can assume more extended conformations, and may be interdigitated between loops (Fig. $4 \mathrm{~h}$ ). In fact, $p$ increased upon heating of the solution $\left(\Delta p=0.5 \mathrm{~nm}, 20 \rightarrow 50^{\circ} \mathrm{C}\right)$ perhaps due to an enhanced molten state of the alkyl chains (Fig. 4f ${ }^{44}$.

Distinct contribution of the monomer subunits. Different supramolecular topologies of the homopolymers of $\mathbf{1}$ and $\mathbf{2}$ indicate that these monomer subunits may contribute differently to the formation of the helicoidal structure. To study this point, we examined the yield of helicoids at different mixing ratios of 1 and 2 while keeping $c_{\mathrm{t}}$ constant at $100 \mu \mathrm{M}$. AFM analyses of the unequal mixtures after equilibration qualitatively demonstrated that helicoids are formed almost exclusively when an excess of 1 is used, whereas fewer were formed when $\mathbf{2}$ is present in excess (Fig. 5a, b and Supplementary Fig. 29). More quantitative data was obtained using SAXS. A plot of the relative intensity of the helicoidal pitch peak $\left(Q=1.15 \mathrm{~nm}^{-1}\right)$ as a function of the $\mathbf{1 : 2}$ ratio showed that the intensity of this peak is considerably stronger for a $6: 4$ mixture (67\% relative to the equimolar mixture) than for a $4: 6$ mixture (17\% relative to the equimolar mixture; Fig. $5 c, d)$. The same trend was observed for the relative absorption intensity at $470 \mathrm{~nm}$, which is diagnostic of the electrostatic interaction between $\mathbf{1}$ and $\mathbf{2}$ (Supplementary Fig. 30). These results demonstrate that $\mathbf{1}$ is the structure-directing monomer subunit in the helicoidal supramolecular copolymerization, as it can form curved aggregates with itself, while $\mathbf{2}$ is an auxiliary monomer subunit that enhances the rosette-rosette interaction via its interaction with $\mathbf{1}$. The decreased helicoid yield when $\mathbf{2}$ is in excess can be attributed to the increased probability of unfavorable self-stacking between 2 moieties.

The distinctly different contributions of $\mathbf{1}$ and $\mathbf{2}$ to the formation of helicoidal structures were further highlighted by experiments using chiral monomers. For that purpose, we synthesized the chiral subunits $\mathbf{1 S}$ and $\mathbf{2 S}$ (Fig. 5e and Supplementary Figs. 2 and 3), albeit that well-defined assemblies were not obtained from individual pure solutions of 15 and $2 S$ (Supplementary Figs. 31 and 32). The sterically demanding chiral chains probably hamper the ordered stacking of their rosettes, as inferred from the observed quick precipitation upon cooling. We therefore investigated mixed systems of chiral and achiral monomers while keeping $c_{\mathrm{t}}$ constant at $500 \mu \mathrm{M}$. As the curvature-forming monomer $\mathbf{1}$ is essential for the formation of helicoids, a 1:1 mixture of $\mathbf{1 S / 2}$ only afforded linear fibers (Supplementary Fig. 33). Even the combination of $\mathbf{1}$ and $\mathbf{1 S}$ in a 1:1:2 mixture of $\mathbf{1 S} / \mathbf{1} / \mathbf{2}$ did not produce helicoidal structures, 


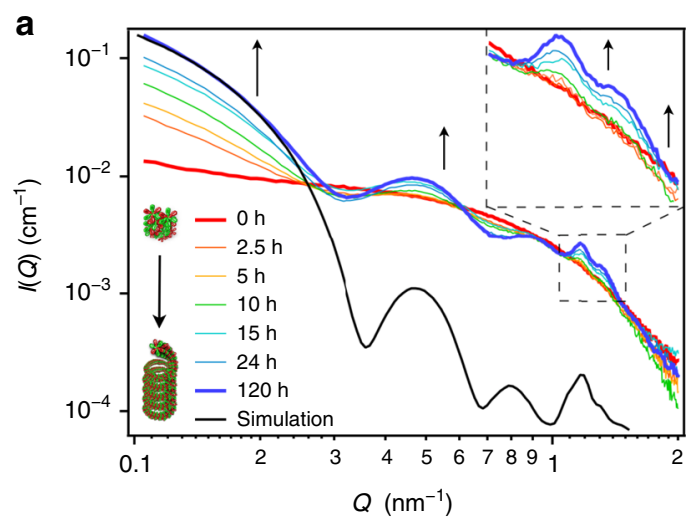

b

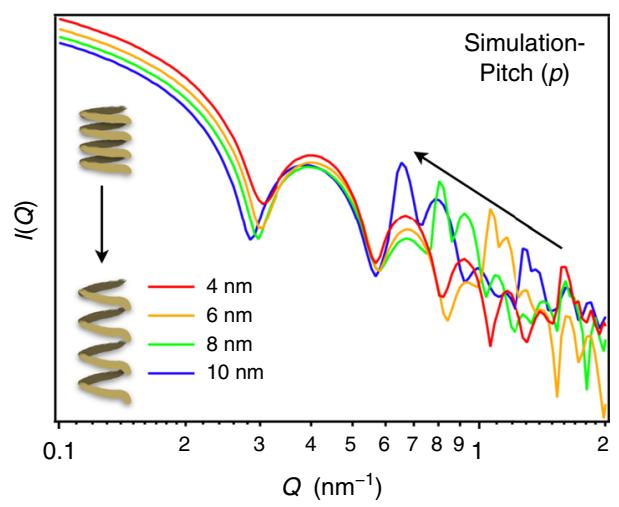

C

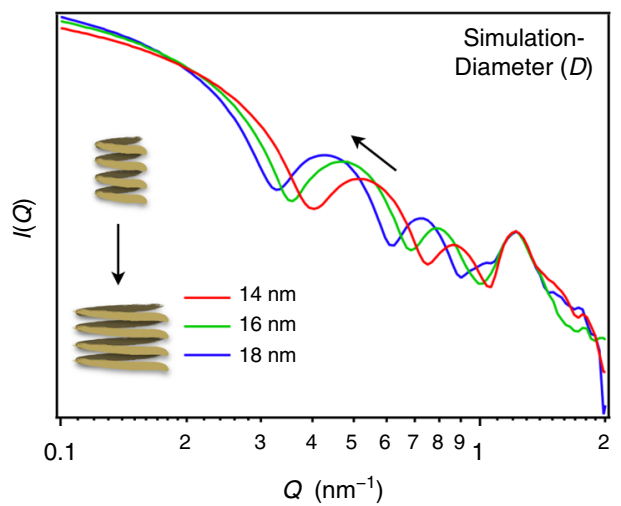

e

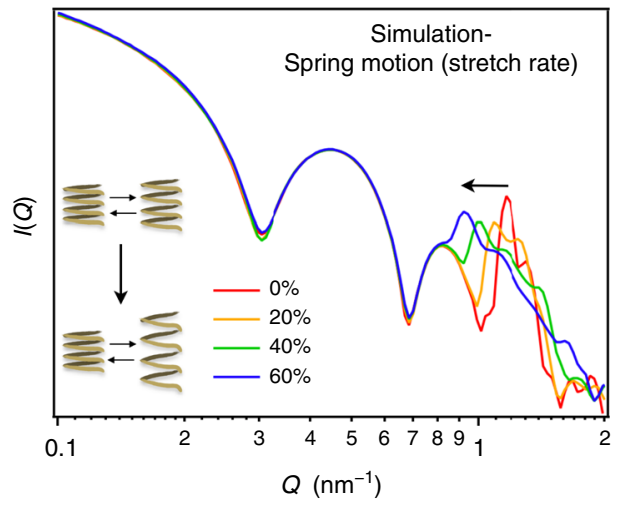

d

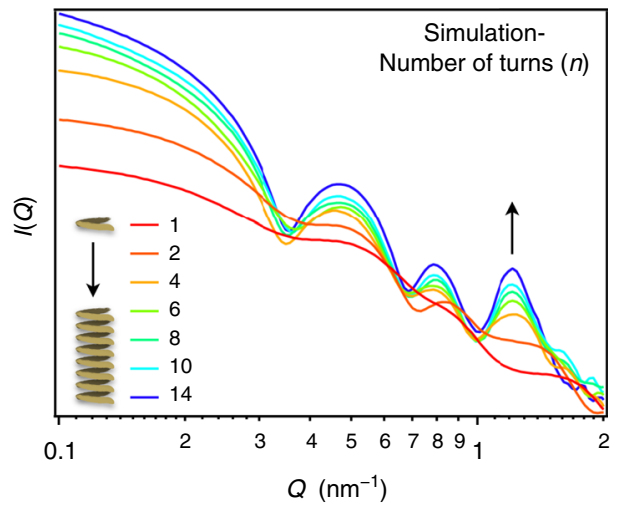

f

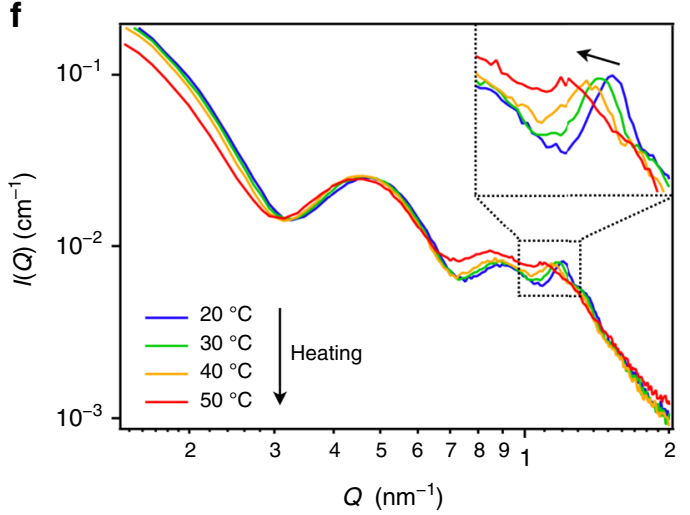

g

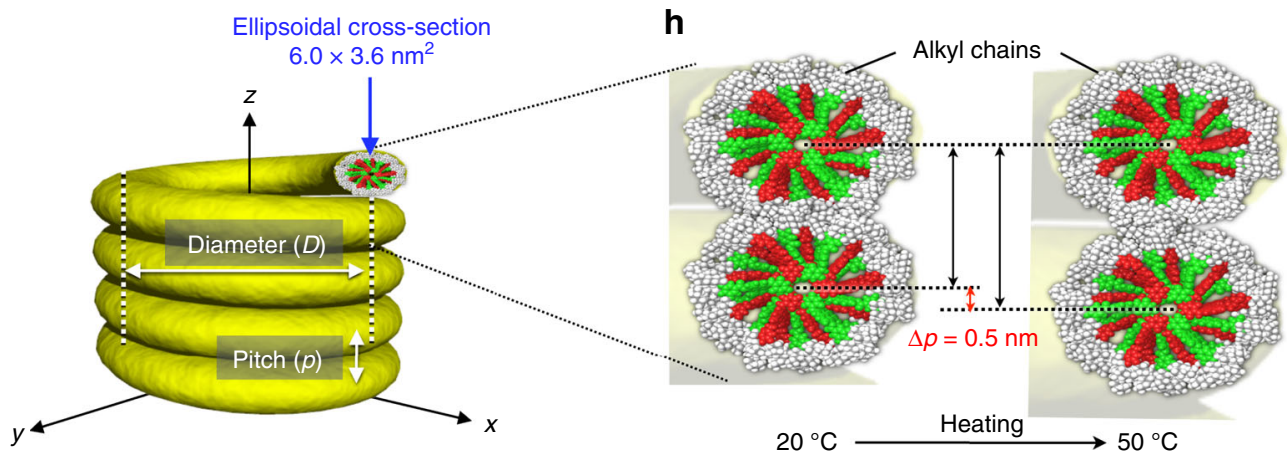

although amorphous coaggregates were afforded kinetically (Supplementary Fig. 34).

In contrast, the incorporation of chiral subunit $2 S$ into the system was found to promote the formation of chiral helicoids. Circular dichroism (CD) measurements demonstrated the timedependent emergence of a Cotton effect for a 1:1 mixture of 1/2S after cooling; the mixture equilibrated after $2 \mathrm{~h}$ and afforded short coils (Supplementary Fig. 35). The Cotton effect observed for 1/2S was entirely dissimilar to that of a 1:1 mixture of $2 / 2 \mathrm{~S}$, suggesting that the chiral substituents of $2 \mathrm{~S}$ also control chiral packing of $\mathbf{1}$ through the alternative stacking of the monomers (Supplementary Fig. 36). Interestingly, when a fraction of $2 S$ was 
Fig. 4 Experimental and simulated SAXS profiles. a Time-dependent SAXS profiles of $\mathbf{1} / \mathbf{2}\left(c_{\mathrm{t}}=500 \mu \mathrm{M}\right)$ during $120 \mathrm{~h}$ of aging. The arrows indicate changes with time. The black solid line represents the simulation result that was most similar to the experimental data, which is based on the following parameters: pitch distance $(p)=5.5 \mathrm{~nm}$, coil center-to-center distance $(D)=16 \mathrm{~nm}$, persistence length defined by the turn number $(n)=14,5 \%$ dispersity, and dynamic motion $(p \pm 10 \%)$ in the $z$-direction. $\mathbf{b}$-e Simulated SAXS profiles showing the effects of changing various parameters: $\mathbf{b} p$ (for $D=16 \mathrm{~nm}, n=$ 14 , and no dynamics); c $D$ (for $p=5.5 \mathrm{~nm}, n=14$, and no dynamics); $\mathbf{d} n$ (for $p=5.5 \mathrm{~nm}, D=16 \mathrm{~nm}$, and no dynamics); e dynamics in the pitch direction (for $p=5.5, D=16 \mathrm{~nm}$, and $n=14$ ). $5 \%$ dispersity in $D$ was included in all the simulations. $\mathbf{f}$ Temperature-dependent SAXS profiles of the helicoid of $\mathbf{1} / \mathbf{2}$ at $20,30,40$, and $50^{\circ} \mathrm{C} . \mathbf{g}$ Schematic representation of the helicoid showing the parameters $D$ and $p$ used for the model fitting of the SAXS data. $\mathbf{h}$ Schematic representation of the relaxing interdigitation of alkyl chains by the heating process.

replaced with achiral 2 , the Cotton effect of the resulting $1 / 2 / 2 S$ at equilibrium became more intense as the fraction of 2 was increased, and reached a maximum at a ratio of 2:1:1 (Fig. 5f, g). The intensity of the Cotton effect of this ternary mixture was twice that of the $\mathbf{1} / \mathbf{2 S}$ binary mixture, despite the reduced content of the chiral monomer $2 \mathrm{~S}$ (i.e., $125 \mu \mathrm{M}$ in $\mathbf{1} / \mathbf{2} / \mathbf{2 S}$ vs. $250 \mu \mathrm{M}$ in $\mathbf{1} / \mathbf{2 S})^{45}$. This optimum ternary mixture afforded elongated helicoidal fiber whose morphology was apparently the same as that of $1 / 2$ (Fig. 5h). Although the helicity of the $1 / 2 / 2 S$ helicoids could not be identified due to their tightly folded structure, this negative Cotton effect strongly suggests the counter clockwise (CCW) rotation of rosettes that leads to $M$-type helicoids (Fig. 5i). The strong correlation between the Cotton effect and helicoidal structure corroborates our previous hypothesis that unidirectional rotation in the rosette stacking leads to the helicoidal growth of a series of supramolecular polymers ${ }^{20}$.

Catastrophic transition. Although the reversibility of monomer binding is one of the major advantages of supramolecular polymers, the dissociation (depolymerization) process is not typically afforded much attention. In the solution state, most supramolecular polymers begin to depolymerize isodesmically upon heating, even if they are polymerized via a nucleation-elongation mechanism ${ }^{46}$. In this context, the thermal behavior of the present helicoids is unique as they abruptly transform into amorphous coaggregates upon heating due to the presence of kinetic coaggregates. Figure $6 \mathrm{a}$ shows the dissociation of the amorphous coaggregates and the helicoids of a 1:1 mixture of 1/2 $\left(c_{\mathrm{t}}=100\right.$ $\mu \mathrm{M})$ as monitored by UV-vis spectroscopy. The cooling and heating curves of the amorphous coaggregates ( $\mathrm{i}$ and ii, respectively) exhibit a small thermal hysteresis, which originates from the progress of the competing supramolecular copolymerization process after cooling (iii). In sharp contrast, the heating curve of the helicoids exhibits a plateau up to $40{ }^{\circ} \mathrm{C}$ (iv), above which it gradually changes and completely overlaps with the cooling curve (i) without thermal hysteresis. When we switched the heating procedure to cooling at $55^{\circ} \mathrm{C}$, the subsequent second cooling curve was identical to the first (i), demonstrating the complete dissociation of the helicoids at $\sim 50{ }^{\circ} \mathrm{C}$ (Supplementary Fig. 37). The aforementioned chiral 2:1:1 ternary mixture of $\mathbf{1} / \mathbf{2} / \mathbf{2 S}$ also displayed a similar thermal response in the $\mathrm{CD}$ measurements: the Cotton effect was abruptly attenuated at $\sim 40{ }^{\circ} \mathrm{C}$ and entirely eliminated at $55^{\circ} \mathrm{C}$ (Fig. 6b).

In the plateau region $\left(20-45^{\circ} \mathrm{C}\right)$, DLS measurements showed only a moderate reduction in $D_{\mathrm{H}}(200 \mathrm{~nm} \rightarrow 157 \mathrm{~nm}$; Fig. $6 \mathrm{c})$. The $D_{\mathrm{H}}$ abruptly decreases from $157 \mathrm{~nm}$ at $45^{\circ} \mathrm{C}$ to $12 \mathrm{~nm}$ at $50^{\circ}$ $\mathrm{C}$, and further to a reported $0.7 \mathrm{~nm}$ at $65^{\circ} \mathrm{C}$, indicating a complete dissociation to the monomeric state. The first abrupt decrease probably corresponds to the helicoids transitioning into amorphous coaggregates. This assertion was further supported by AFM and SAXS measurements (Fig. 6d, e and Supplementary Fig. 38).

The phase transition from helicoids to amorphous coaggregates suggests that the Gibbs free energy changes $\left(\Delta G^{\circ}\right)$ of the helicoids $\left(\Delta G^{\circ}{ }_{\text {hel }}\right)$ and the amorphous coaggregates $\left(\Delta G_{\text {amo }}^{\circ}\right)$ should intersect as the temperature is increased, due to the increasing contribution of entropy ${ }^{47}$. We therefore attempted to estimate the critical temperature $\left(T_{\mathrm{c}}\right)$ at which $\Delta G_{\text {amo }}^{\circ}=\Delta G_{\text {hel }}^{\circ}$ (Fig. 6f). The $\Delta G^{\circ}$ values of the two species were estimated from temperature-dependent absorption measurements at different $c_{\mathrm{t}}$. To observe the direct thermal dissociation of the helicoids into monomers, we diluted the solution to $c_{\mathrm{t}}=10 \mu \mathrm{M}$, by which the onset of aggregation is expected to be sufficiently lower than the $T_{c}$. By this dilution, a sigmoidal dissociation of helicoids into monomers was observed with the onset of aggregation at $33^{\circ} \mathrm{C}$, suggesting dissociation via an isodesmic mechanism (Fig. $6 \mathrm{~g}$ ). A van't Hoff analysis of the isodesmic curve gave $\Delta H^{\circ}=-96 \mathrm{~kJ} \mathrm{~mol}^{-1}$ and $\Delta S^{\circ}=-218 \mathrm{~J} \mathrm{~mol}^{-1} \mathrm{~K}^{-1}$ (Supplementary Fig. 39 and Supplementary Table 1). As described above, we obtained cooperative cooling curves for the amorphous coaggregates, from which we estimated values of $\Delta H^{\circ}=-57 \mathrm{~kJ} \mathrm{~mol}^{-1}$ and $\Delta S^{\circ}=$ $-93 \mathrm{~J} \mathrm{~mol}^{-1} \mathrm{~K}^{-1}$ using van't Hoff analysis. The larger negative entropy change of the helicoids clearly reflects the eventual conversion of the diverse rosette complexes into one alternating configuration (1:2:1:2:1:2:). This large entropic penalty could be compensated by the enthalpy change of $-96 \mathrm{~kJ} \mathrm{~mol}^{-1}$ in the helicoidal organization, i.e., the interaction between loops as well as the electrostatic interaction between 1 and 2. From these $\Delta H^{\circ}$ and $\Delta S$ ${ }^{\circ}$ values, the $T_{\mathrm{c}}$ at which $\Delta G^{\circ}$ amo $=\Delta G^{\circ}$ hel was calculated to be $42{ }^{\circ} \mathrm{C}$ (Fig. 6f). This value can be rationalized by the observations that the helicoidal copolymerization proceeded when the temperature was kept at $35^{\circ} \mathrm{C}$ during the cooling process, and the transition to the amorphous coaggregates proceeded when the temperature was kept at $45^{\circ} \mathrm{C}$ during the heating process $\left(c_{\mathrm{t}}=100 \mu \mathrm{M}\right.$; Fig. $\left.6 \mathrm{~h}\right)$. Accordingly, the relative thermodynamic stability of the two species switches at $\sim 40{ }^{\circ} \mathrm{C}$, and the two processes are in equilibrium. Above $50^{\circ} \mathrm{C}$, however, the helicoid cannot form due to the entropic contribution, and therefore the heating curve coincided with the cooling curve.

\section{Discussion}

Based on our hierarchical system for the construction of topological supramolecular polymers using hydrogen-bonded rosette complexes, we have presented a supramolecular copolymerization of two structurally near-identical monomer subunits. Due to the small structural difference between the two monomers, they kinetically coassemble to form various hydrogen-bonded complexes that associate in a disorderly manner into amorphous coaggregates. The weak electrostatic interactions between the two electronically complementary monomers drive the integration of the various complexes into one specific configuration through hydrogen bond rearrangement, which results in their supramolecular copolymerization into helicoidal superstructures. Notably, when homogeneous solutions of each of the monomers are prepared, one monomer predominantly forms thermodynamically stable toroidal assemblies, whereas the other can only afford illdefined aggregates. In this context, we have successfully achieved the topological extension of toroidal to helicoidal architectures via supramolecular copolymerization. Although the helicoidal structures have a clear enthalpic advantage over amorphous coaggregates, the large entropic penalties associated with the 

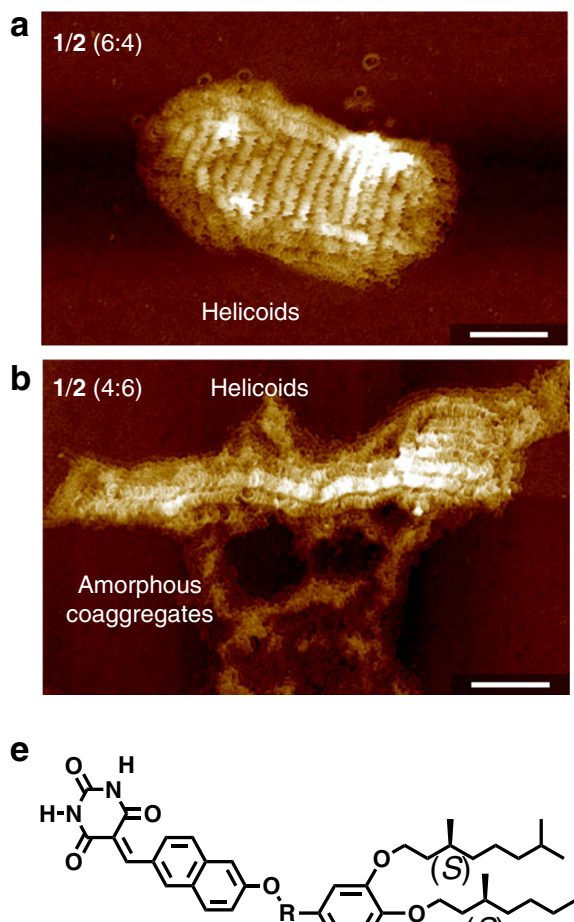

Chiral subunits 1S: $\mathrm{R}=\mathrm{CH}_{2}=$ 2S: $\mathrm{R}=\mathrm{CO}=$

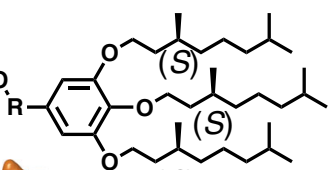

(S)
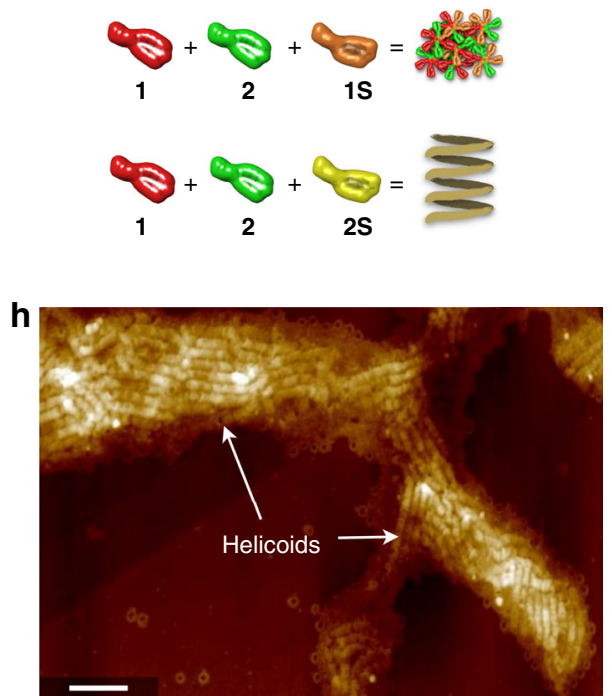

C

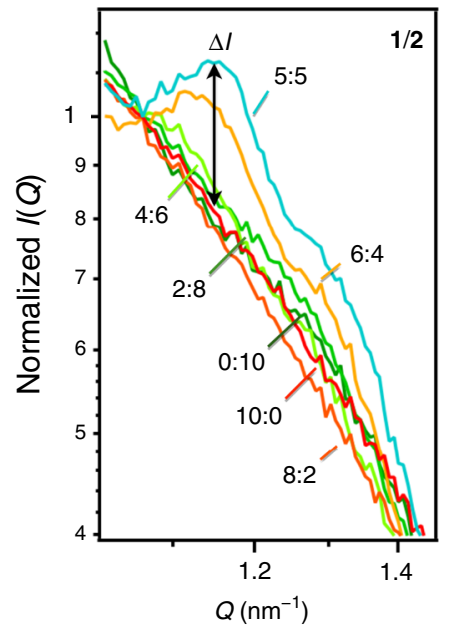

f

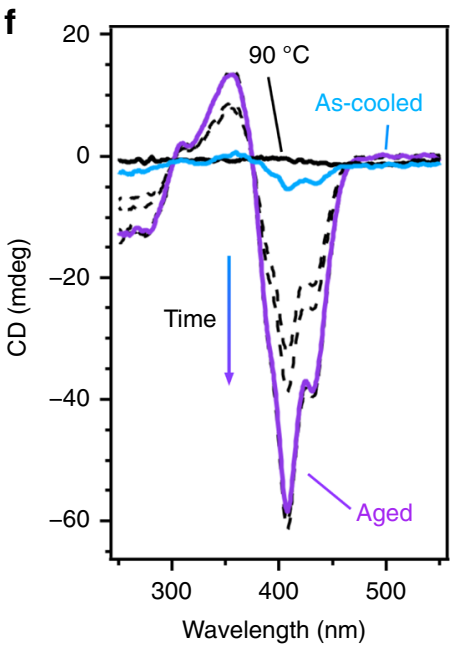

d

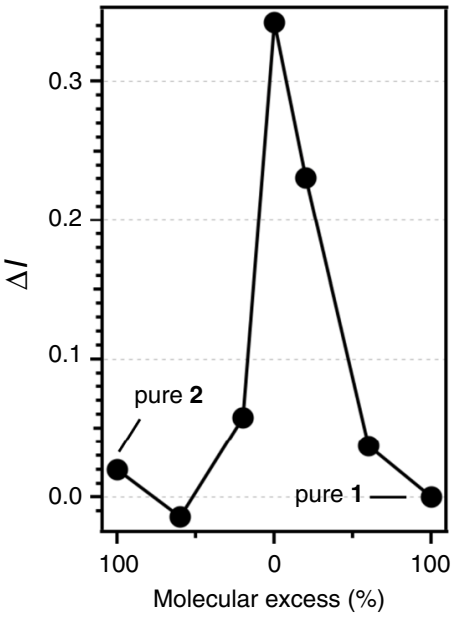

g

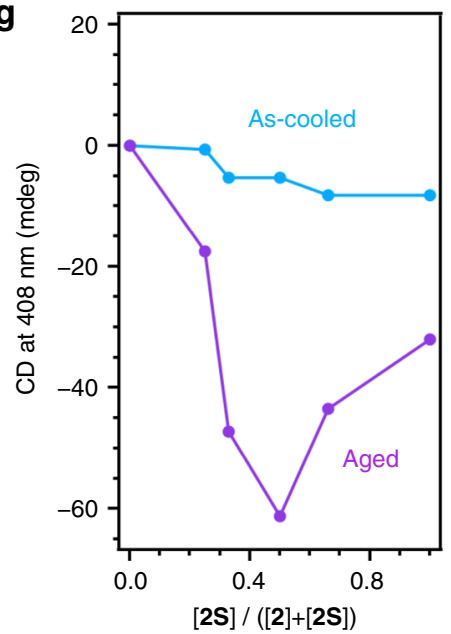

i

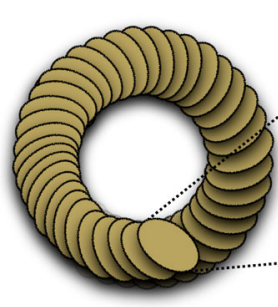

$P$-helicoid

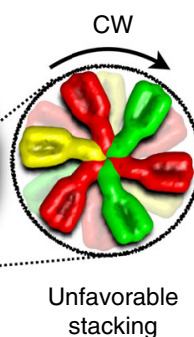

stacking

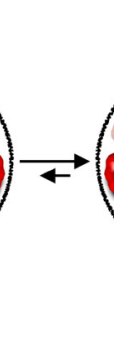

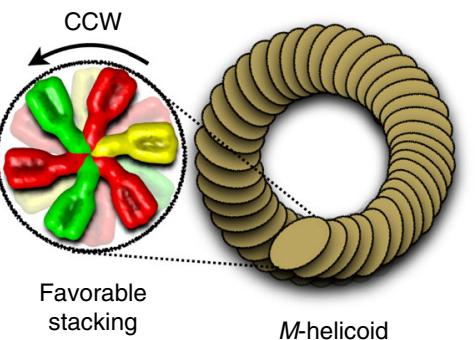

Fig. 5 Distinct contributions of the different monomers. a, b AFM images of $\mathbf{1} / \mathbf{2}(6: 4 ; \mathbf{a})$ and $\mathbf{1} / \mathbf{2}(4: 6 ; \mathbf{b})$ at $c_{t}=100 \mu M$. The samples were prepared by

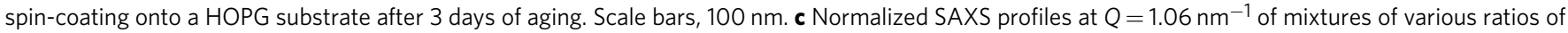
1 and 2. Ratios: 1/2 (10:0; red), (8:2; dark orange), (6:4; orange), (5:5; blue), (4:6; light green), (2:8; green), and (0:10; dark green). d Plots of $\Delta /$ estimated from SAXS profiles as a function of the molecular excess. e Molecular structures of $\mathbf{1 S}$ and $\mathbf{2 S}$ as well as schematic representations of their tertiary mixtures. $\mathbf{f} C D$ spectra of $\mathbf{1 / 2} \mathbf{2} \mathbf{S}\left(2: 1: 1 ; c_{t}=500 \mu \mathrm{M}\right)$ measured at $90^{\circ} \mathrm{C}$ (black), immediately after cooling (blue), and after 9 days of aging (purple). $\mathbf{g}$ Plots of the intensity of the CD spectra at $408 \mathrm{~nm}$ immediately after cooling (blue) and after aging until reaching equilibrium (purple) as a function of the ratio of $\mathbf{2 S}$ relative to the total amount of $\mathbf{2}$ derivatives; the concentration of $\mathbf{1}(250 \mu \mathrm{M})$ and the $c_{t}(500 \mu \mathrm{M})$ were kept constant. $\mathbf{h}$ AFM image of the helicoids of $\mathbf{1} / \mathbf{2} / \mathbf{2} \mathbf{S}\left(2: 1: 1 ; c_{t}=500 \mu \mathrm{M}\right)$ after 7 days of aging. Scale bar, $100 \mathrm{~nm}$. $\mathbf{i}$ Proposed mechanism for the elongation of the unidirectional helicoidal fiber.

alternating molecular arrays at both the hydrogen-bonded complex level and the hierarchical stacking level led to catastrophic transition behavior. All of these unique outcomes further highlight the advantages of designing supramolecular polymers in a hierarchical manner, which has rarely been achieved in artificial supramolecular polymers, but is common in their natural counterparts. As demonstrated in this study, the hierarchical approach enables the integration of a molecular recognition process to preorganize the supramolecular building blocks, which is distinctly different from a supramolecular polymerization directly 

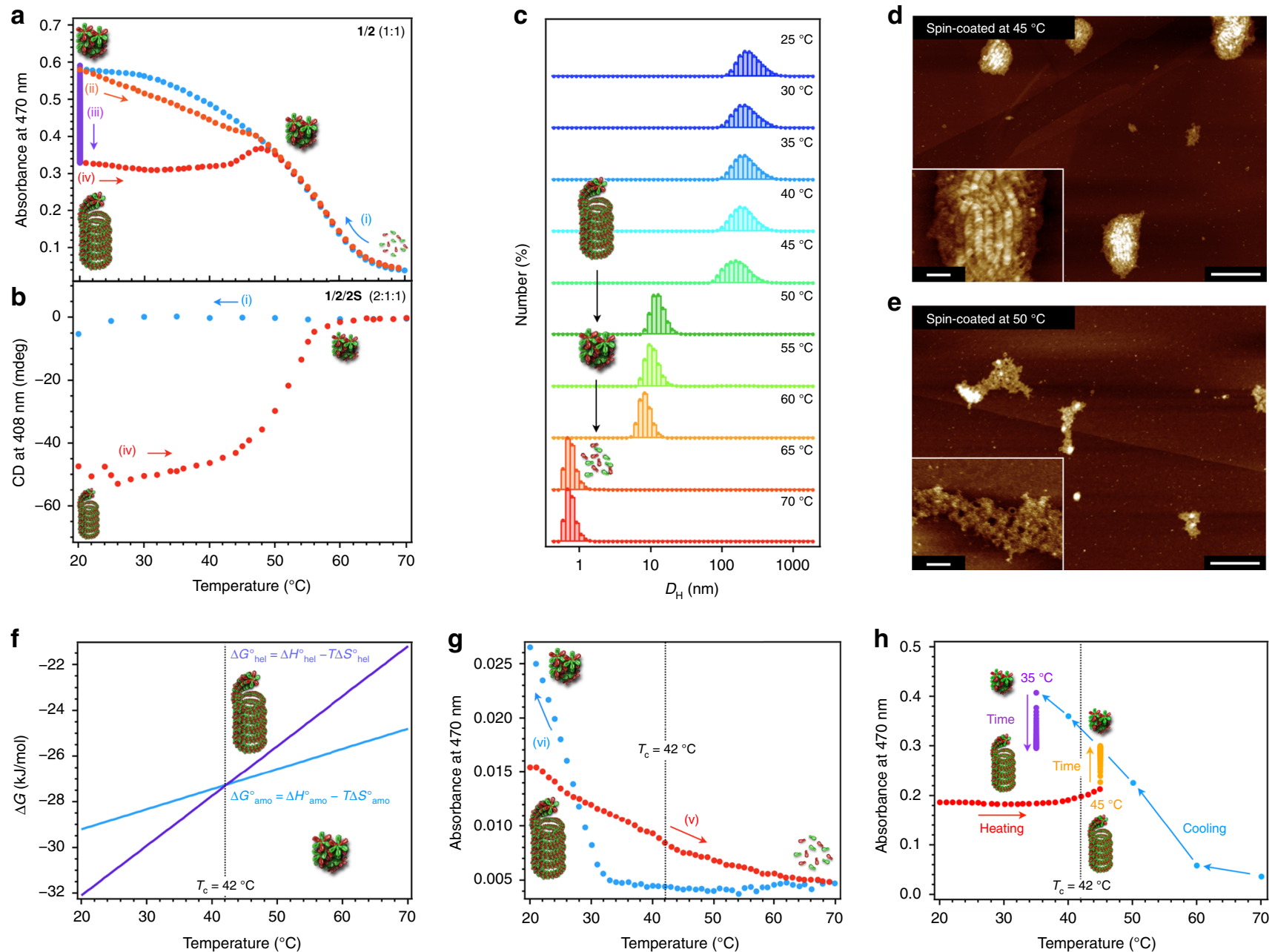

Fig. 6 Catastrophic transition. a, b Plots of absorbance at $470 \mathrm{~nm}(\mathbf{a})$ and $C D$ intensity at $408 \mathrm{~nm}(\mathbf{b})$ as a function of temperature for $\mathbf{1 / 2}\left(1: 1 ; c_{t}=100\right.$ $\mu \mathrm{M})$ and $\mathbf{1} / \mathbf{2} / \mathbf{2 S}\left(2: 1: 1 ; c_{t}=500 \mu \mathrm{M}\right)$ in the first cooling process (i; blue), in the heating process immediately after cooling (ii; orange), in the aging process (iii; purple), and in the heating process after aging (iv; red). Heating and cooling rate is $1^{\circ} \mathrm{C} \mathrm{min}^{-1}$. c Temperature-dependent DLS profiles of the helicoids of $\mathbf{1 / 2}\left(1: 1 ; c_{t}=100 \mu \mathrm{M}\right)$ from 25 to $70^{\circ} \mathrm{C}$. d, e AFM images of $\mathbf{1} / \mathbf{2}\left(1: 1 ; c_{t}=100 \mu \mathrm{M}\right)$ samples prepared by spin-coating at $45^{\circ} \mathrm{C}(\mathbf{d})$ and $50^{\circ} \mathrm{C}(\mathbf{e})$. Scale bars, $300 \mathrm{~nm}$ and $50 \mathrm{~nm}$ (inset). f Phase diagram estimated from $\Delta G=\Delta H-T \Delta S$. $\mathbf{g}$ Plots of the spectral changes of the absorbance at $470 \mathrm{~nm}$ as a function of the temperature for $\mathbf{1} / \mathbf{2}\left(1: 1 ; c_{t}=10 \mu \mathrm{M}\right)$ during the heating ( $\mathrm{v}$; red) and cooling (vi; blue) processes. $\mathbf{h}$ Blue and purple dots: plots of absorbance at $470 \mathrm{~nm}$ as a function of temperature for $\mathbf{1 / 2}\left(1: 1 ; c_{t}=100 \mu \mathrm{M}\right.$, amorphous coaggregate) in the first cooling process (blue) and subsequent aging process at $35^{\circ} \mathrm{C}$ $(10 \mathrm{~h})$. Red and orange dots: plots of absorbance at $470 \mathrm{~nm}$ as a function of temperature for the equilibrated $\mathbf{1} / \mathbf{2}\left(1: 1 ; c_{\mathrm{t}}=100 \mu \mathrm{M}\right.$, helicoid) in the heating process (red) and subsequent aging at $45^{\circ} \mathrm{C}(10 \mathrm{~h})$.

driven by host-guest systems. We are currently exploring further complex nanosystems featuring not only structural but also functional biomimicry, and the result of these studies will be reported in due course.

\section{Methods}

General. Column chromatography was performed using $63-210 \mu \mathrm{m}$ silica gel. All other commercially available reagents and solvents were of reagent grade and used without further purification. The solvents for the preparation of the assemblies were all spectral grade and used without further purification. ${ }^{1} \mathrm{H}$ NMR spectra were recorded on Bruker DPS300 or JEOL JNM-ECA500 NMR spectrometers and chemical shifts are reported in parts per million (ppm) with the signal of TMS as internal standard. Electrospray ionization MS spectra were measured on an Exactive (Thermo Scientific). UV-vis and fluorescent spectra were recorded on a JASCO V660 spectrophotometer and a JASCO FP6600 spectropolarimeter, respectively. Both the spectrometers are equipped with Peltier device temperaturecontrol unit. DLS measurements were performed on a Zetasizer Nano S (Malvern Instruments) device using non-invasive back-scatter technology (NIBS) under 4.0 $\mathrm{mW}$ He-Ne laser $(633 \mathrm{~nm})$. The scattering angle was set at $173^{\circ}$. Fourier transform IR (FT-IR) spectra were measured on JASCO FT/IR-4600 spectrometer.
Materials. Compound 1S, 2, and 2S were synthesized according to the section of synthesis and characterization data in Supplementary materials. $\mathbf{1}$ and $\mathbf{3}$ were synthesized according to our previous report ${ }^{23}$. All starting materials and reagents were purchased from commercial suppliers and used without further purification. Air sensitive reactions were conducted under nitrogen atmosphere using dry solvents.

Atomic force microscopy (AFM). AFM images were acquired under ambient conditions using Multimode 8 Nanoscope V microscope (Bruker Instruments) in peak force tapping (Scanasyst) mode. Silicon cantilevers (SCANASYST-AIR) with a spring constant of $0.4 \mathrm{~N} / \mathrm{m}$ and frequency of $70 \mathrm{kHz}$ (nominal value, Bruker, Japan) were used. Samples were prepared by spin-coating assembly solutions onto freshly cleaved HOPG.

Small angle X-ray scattering (SAXS). SAXS experiments were performed at BL10C of the Photon Factory of the High Energy Accelerator Research Organization (KEK) in Tsukuba, Japan ${ }^{48}$. Sample solutions were placed into $1.25-\mathrm{mm}$ path length cells $(20-\mu \mathrm{m}$ thickness quartz glass windows surrounded by stainless steel), and the temperature was fixed at $293 \mathrm{~K}$. X-ray with a wavelength of $1.5 \AA$ and a sample-detector distance of $1029 \mathrm{~mm}$ (calibrated with silver behenate) resulted in a detectable $Q$ range in the order of $0.1-5.9 \mathrm{~nm}^{-1}$. Sixty frames were collected with 
each exposure time of $10 \mathrm{~s}$. Because no radiation damage was observed, the collected data were averaged to give a total integration time of $600 \mathrm{~s}$. The $2 \mathrm{D}$ scattering data (detector: DECTRIS PILATUS3 2M) were radially averaged to yield $1 \mathrm{D}$ scattering intensity data $[I(Q)$ vs. $Q]$. These data were then normalized using water as a reference, and the following subtraction of the background (quartz glass windows and solvent) gave absolute scattering intensity $I(Q)$ in $\mathrm{cm}^{-1}$. All data reduction was performed using the software package SAngler ${ }^{49}$. In temperaturedependent SAXS experiments, the temperature of sample solution was controlled using a HCS302-LN190 (Instec Inc.). Sample solutions were placed in quartz capillaries ( $2 \mathrm{~mm}$ in diameter and $10 \mu \mathrm{m}$ in wall thickness), and the top of the capillary was sealed with resin to avoid evaporation of the solvent at higher temperature. SAXS data were collected using X-ray with a wavelength of $1 \AA$.

\section{Data availability}

All data needed to evaluate the conclusions in the paper are present in the paper and/or the Supplementary Materials. Additional data related to this paper may be requested from the corresponding authors.

Received: 15 December 2019; Accepted: 9 March 2020;

Published online: 01 April 2020

\section{References}

1. Pieters, B. J. G. E., van Eldijk, M. B., Nolte, R. J. M. \& Mecinovic, J. Natural supramolecular protein assemblies. Chem. Soc. Rev. 45, 24-39 (2016).

2. Wei, G., Xi, W., Nussinov, R. \& Ma, B. Protein ensembles: How does nature harness thermodynamic fluctuations for life? The diverse functional roles of conformational ensembles in the cell. Chem. Rev. 116, 6516-6551 (2016).

3. Durham, A. C. H. \& Klug, A. Polymerization of tobacco mosaic virus protein and its control. Nat. N. Biol. 229, 42-46 (1971).

4. Fowler, D. M., Koulov, A. V., Balch, W. E. \& Kelly, J. W. Functional amyloidfrom bacteria to humans. Trends Biochem. Sci. 32, 217-224 (2007).

5. Fletcher, D. A. \& Mullins, R. D. Cell mechanics and the cytoskeleton. Nature 463, 485-492 (2010).

6. Huber, F. et al. Emergent complexity of the cytoskeleton: from single filaments to tissue. Adv. Phys. 62, 1-112 (2013).

7. von der Ecken, J. et al. Structure of the F-actin-tropomyosin complex. Nature 519, 114-117 (2015).

8. Nogales, E., Wolf, S. G. \& Downing, K. H. Structure of the alpha beta tubulin dimer by electron crystallography. Nature 391, 199-203 (1998).

9. Gigant, B. et al. The 4 A X-ray structure of a tubulin:stathmin-like domain complex. Cell 102, 809-816 (2000).

10. Dutcher, S. K. The tubulin fraternity: alpha to eta. Curr. Opin. Cell Biol. 13, 49-54 (2001)

11. Wade, R. H. On and around microtubules: an overview. Mol. Biotechnol. 43, 177-191 (2009).

12. Buey, R. M., Díaz, J. F. \& Andreu, J. M. The nucleotide switch of tubulin and microtubule assembly: a polymerization-driven structural change. Biochemistry 45, 5933-5938 (2006).

13. de Greef, T. F. A. \& Meijer, E. W. Materials science: supramolecular polymers. Nature 453, 171-173 (2008).

14. Jain, A. \& George, S. J. New directions in supramolecular electronics. Mater. Today 18, 206-214 (2015).

15. Aida, T., Meijer, E. W. \& Stupp, S. I. Functional supramolecular polymers. Science 335, 813-817 (2012).

16. Sorrenti, A., Leira-Iglesias, J., Markvoort, A. J., de Greef, T. F. A. \& Hermans, T. M. Non-equilibrium supramolecular polymerization. Chem. Soc. Rev. 46, 5476-5490 (2017).

17. Mattia, E. \& Otto, S. Supramolecular systems chemistry. Nat. Nanotech. 10, 111-119 (2015).

18. Yagai, S. Supramolecularly engineered functional $\pi$-assemblies based on complementary hydrogen-bonding interactions. Bull. Chem. Soc. Jpn. 88, 28-58 (2015).

19. Adhikari, B. et al. Hydrogen-bonded rosettes comprising $\pi$-conjugated systems as building blocks for functional one-dimensional assemblies. Chem. Commun. 53, 9663-9683 (2017).

20. Yagai, S., Kitamoto, Y., Datta, S. \& Adhikari, B. Supramolecular polymers capable of controlling their topology. Acc. Chem. Res. 52, 1325-1335 (2019).

21. Adhikari, B. et al. Light-induced unfolding and refolding of supramolecular polymer nanofibres. Nat. Commun. 8, 15254 (2017).

22. Prabhu, D. D. et al. Self-folding of supramolecular polymers into bioinspired topology. Sci. Adv. 4, eaat8466 (2018).

23. Yagai, S. et al. Self-organization of hydrogen-bonding naphthalene chromophores into J-type nanorings and H-type nanorods: impact of regioisomerism. Angew. Chem. Int. Ed. 51, 6643-6647 (2012).
24. Hollamby, M. J. et al. Simultaneous SAXS and SANS analysis for the detection of toroidal supramolecular polymers composed of noncovalent supermacrocycles in solution. Angew. Chem. Int. Ed. 55, 9890-9893 (2016).

25. Aratsu, K. et al. Self-sorting regioisomers through the hierarchical organization of hydrogen-bonded rosettes. Chem. Commun. 52, 8211-8214 (2016).

26. Aratsu, K. \& Yagai, S. Self-sorting of rosette-forming naphthalene barbiturates into distinct toroidal assemblies. ChemPlusChem 84, 619-622 (2019).

27. Kitamoto, Y. et al. One-shot preparation of topologically chimeric nanofibers via a gradient supramolecular copolymerization. Nat. Commun. 10, 4578 (2019).

28. Taylor, P. N. \& Anderson, H. L. Cooperative self-assembly of double-strand conjugated porphyrin ladders. J. Am. Chem. Soc. 121, 11538-11545 (1999).

29. Wu, A. \& Isaacs, L. Self-sorting: the exception or the rule? J. Am. Chem. Soc 125, 4831-4835 (2003).

30. Safont-Sempere, M. M., Fernández, G. \& Würthner, F. Self-sorting phenomena in complex supramolecular systems. Chem. Rev. 111, 5784-5814 (2011).

31. Chakraborty, S., Kar, H., Sikder, A. \& Ghosh, S. Steric ploy for alternating donor-acceptor co-assembly and cooperative supramolecular polymerization. Chem. Sci. 8, 1040-1045 (2017).

32. Hirao, T., Kudo, H., Amimoto, T. \& Haino, T. Sequence-controlled supramolecular terpolymerization directed by specific molecular recognitions. Nat. Commun. 8, 634 (2017).

33. Ogoshi, T. et al. Ring shape-dependent self-sorting of pillar[n] arenes assembled on a surface. Commun. Chem. 1, 92 (2018).

34. Görl, D., Zhang, X., Stepanenko, V. \& Würthner, F. Supramolecular block copolymers by kinetically controlled co-self-assembly of planar and coretwisted perylene bisimides. Nat. Commun. 6, 7009 (2015).

35. Jonkheijm, P., van der Schoot, P., Schenning, A. P. H. J. \& Meijer, E. W. Probing the solvent-assisted nucleation pathway in chemical self-assembly. Science 313, 80-83 (2006).

36. Smulders, M. M. J. et al. How to distinguish isodesmic from cooperative supramolecular polymerisation. Chem. Eur. J. 16, 362-367 (2010).

37. Shen, B. et al. Autonomous helical propagation of active toroids with mechanical action. Nat. Commun. 10, 1080 (2019).

38. Korevaar, P. A. et al. Pathway complexity in supramolecular polymerization. Nature 481, 492-496 (2012).

39. Ogi, S., Sugiyasu, K., Manna, S., Samitsu, S. \& Takeuchi, M. Living supramolecular polymerization realized through a biomimetic approach. Nat Chem. 6, 188-195 (2014).

40. Ogi, S., Stepanenko, V., Thein, J. \& Würthner, F. Impact of alkyl spacer length on aggregation pathways in kinetically controlled supramolecular polymerization. J. Am. Chem. Soc. 138, 670-678 (2016)

41. Jiang, W. \& Schalley, C. A. Molecular recognition and self-assembly special feature: integrative self-sorting is a programming language for high level selfassembly. Proc. Natl Acad. Sci. USA 106, 10425-10429 (2009).

42. He, Z., Jiang, W. \& Schalley, C. A. Integrative self-sorting: a versatile strategy for the construction of complex supramolecular architecture. Chem. Soc. Rev. 44, 779-789 (2015).

43. Ogi, S., Fukui, T., Jue, M. L., Takeuchi, M. \& Sugiyasu, K. Kinetic control over pathway complexity in supramolecular polymerization through modulating the energy landscape by rational molecular design. Angew. Chem. Int. Ed. 53, 14363-14367 (2014).

44. Kouwer, P. H. J. \& Mehl, G. H. Hierarchical organisation in shapeamphiphilic liquid crystals. J. Mater. Chem. 19, 1564-1575 (2009).

45. Ajayaghosh, A., Varghese, R., George, S. J. \& Vijayakumar, C. Transcription and amplification of molecular chirality to oppositely biased supramolecular $\pi$ helices. Angew. Chem. Int. Ed. 45, 1141-1144 (2006).

46. de Greef, T. F. A. et al. Supramolecular polymerization. Chem. Rev. 109, 5687-5754 (2009).

47. Tokoro, H. et al. Theoretical prediction of a charge-transfer phase transition. Sci. Rep. 8, 63 (2018)

48. Shimizu, N. et al. BL-10C, the small-angle $\mathrm{x}$-ray scattering beamline at the photon factory. AIP Conf. Proc. 2054, 060041 (2019).

49. Igarashi, N. et al. Upgrade of the small angle X-ray scattering beamlines at the Photon Factory. J. Phys. 272, 012026 (2011).

\section{Acknowledgements}

This work was supported by KAKENHI grant no. 26102010 and a Grant-in-Aid for Scientific Research on Innovative Areas " $\pi$-Figuration" (grant no. 26102001) from the Japanese Ministry of Education, Culture, Sports, Science, and Technology (MEXT). This work was performed with the approval of the Photon Factory Program Advisory Committee (proposal no. 2016G550). S.Y. acknowledges financial support from the Murata Science Foundation. K.A. thanks the JSPS for a research fellowship (17J02520). We thank Dr. Tomonori Ohba for assisting with TEM measurements. 


\section{Author contributions}

S.Y. and K.A. designed the project. K.A. synthesized 1-3, and performed most of the experimental works except for $1 S$ and 2 S. R.T. synthesized $1 S$ and $2 S$, and carried out the experimental works of them. N.S., H.T., R.H., and S.-i.A. collected the SAXS data. M.J.H. and B.R.P. simulated the SAXS data and wrote SAXS section of the paper. S.Y. and K.A. prepared the overall paper including figures. All authors including Y.K. have contributed by commenting on the paper. The overall project was directed by S.Y.

\section{Competing interests}

The authors declare no competing interests.

\section{Additional information}

Supplementary information is available for this paper at https://doi.org/10.1038/s41467020-15422-6.

Correspondence and requests for materials should be addressed to B.R.P., M.J.H. or S.Y.

Peer review information Nature Communications thanks Luis Sanchez and the other, anonymous, reviewer(s) for their contribution to the peer review of this work. Peer reviewer reports are available.
Reprints and permission information is available at http://www.nature.com/reprints

Publisher's note Springer Nature remains neutral with regard to jurisdictional claims in published maps and institutional affiliations.

\section{(c) (i)}

Open Access This article is licensed under a Creative Commons Attribution 4.0 International License, which permits use, sharing, adaptation, distribution and reproduction in any medium or format, as long as you give appropriate credit to the original author(s) and the source, provide a link to the Creative Commons license, and indicate if changes were made. The images or other third party material in this article are included in the article's Creative Commons license, unless indicated otherwise in a credit line to the material. If material is not included in the article's Creative Commons license and your intended use is not permitted by statutory regulation or exceeds the permitted use, you will need to obtain permission directly from the copyright holder. To view a copy of this license, visit http://creativecommons.org/ licenses/by/4.0/.

(C) The Author(s) 2020 